\title{
Simulation on solar type II radio bursts associated with corona mass ejections
}

\author{
J. I. Sakai, T. Mori, and S. Saito
}

Laboratory for Plasma Astrophysics, Faculty of Engineering, Toyama University, 3190 Gofuku, Toyama, 930-8555, Japan

e-mail: sakaijun@eng.toyama-u.ac.jp

Received 10 March 2005 / Accepted 15 June 2005

\section{ABSTRACT}

The wave emission process of solar type II radio bursts associated with Coronal Mass Ejections (CME) is investigated by using twodimensional, electromagnetic, relativistic Particle-In-Cell (PIC) code. Two simulation results are shown: the first case where the CME propagates perpendicular to the local magnetic field, and the second case where the CME propagates obliquely to the local magnetic field showing two cases of the propagation angle of 80 and 40 degrees. We show that a fast magnetosonic shock wave is formed with both protons and electrons accelerated by the surfatron acceleration mechanism. From the first case we show that the electron Bernstein waves are generated near the shock front and can be converted to extra-ordinary electromagnetic waves (type II radio bursts) through direct linear mode conversion. It is found from the second case that the electrostatic Langmuir waves (Z-mode) are generated near the shock front and can be also converted in extra-ordinary electromagnetic waves through direct linear mode conversion.

Key words. acceleration of particules - plasmas - shock waves - radiation mechanisms: general

\section{Introduction}

The origin of the shock waves in the solar corona that manifest themselves as solar type II radio bursts is one of the most important subjects of solar and solar-terrestrial physics. A general picture of emission from type II shocks suggests that the emission mechanism is plasma emission near the fundamental and second harmonic (Smith 1970; Melrose 1980). Langmuir waves are produced by beams produced in this shock. The excited Langmuir waves may be converted into escaping radio waves by nonlinear wave-wave processes (see reviews by Goldman \& Smith 1986; Cairns et al. 2003; Aschwanden 2004). Recent observations from the Ulysses spacecraft (Simnett 2003) have measured the energy spectrum of the ambient ions associated with the passage of a fast magnetosonic quasi-perpendicular shock driven by a CME. Near the shock front the energy spectrum of the accelerated ions, which are predominantly protons, exhibit features (maxima) at about $250 \mathrm{keV}-1.5 \mathrm{MeV}$. Simnett et al. (2004) show that the protons with energy of about $1 \mathrm{MeV}$, which corresponds to $\left(m_{\mathrm{i}} / m_{\mathrm{e}}\right) V_{\mathrm{A}}\left(M_{\mathrm{A}}-1\right)^{3 / 2}$, can be accelerated by the surfatron mechanism (Sagdeev 1996) of a fast magnetosonic shock wave propagating almost perpendicular to an ambient magnetic field. The in situ measurements therefore illustrate directly that this mechanism is very probably responsible for interplanetary particle acceleration. The shock is also accompanied by an increase in the near-relativistic electron intensity of almost two orders of magnitude.
High-energy solar protons ( $>100 \mathrm{MeV}$ ) associated with CMEs are thought to be the result of the first-order Fermi acceleration process (Fermi 1949; Lee 1983), at a shock wave driven by a solar eruption. The idea of diffusive shock acceleration did not receive much attention until the late 1970s, when Bell (1978) and Blanford \& Ostriker (1978) recognized that a discontinuity (the shock) can accelerate particles rather efficiently. These theories have been debated within the comunity (e.g., Bulanov \& Sokolov 1984; Reames 1999, 2002) because very little is known about the dynamical properties of CMEdriven shock waves after the onset of the eruption and about whether or not the level of turbulence at the shock is sufficient for this mechanism to work. Recently Sokolov et al. (2004) investigated this problem and showed that the diffusive shock acceleration mechanism can account for the production of highenergy sola protons observed during the early stage of gradual events. Since the surfatron mechanism is capable of accelerating protons to $\sim \mathrm{MeV}$ energies, it may provide a solution for the injection problem of the shock acceleration mechanism.

In the present paper we address the formation of shock waves driven by CME, acceleration process of both protons and electrons and the emission mechanism of electromagnetic waves (solar type II bursts) from the shock front, by using a two-dimensional, fully relativistic particle-in cell (PIC) simulation. We did many simulations by changing the shock Alfvén Mach number from $M_{\mathrm{A}}=1.5$ to $M_{\mathrm{A}}=3.0$ and also by changing the propagation angle of the shock from 90 to 40 degrees. It is shown that for fast magnetosonic shock waves 
with $M_{\mathrm{A}} \geq 2.0$ and propagation angle between 90 and 70 degrees protons near the shock front can be accelerated by the surfatron acceleration mechanism, while the emission of the electromagnetic waves from the shock front was observed almost independently of the Alfvén Mach and the propagation angles. Here only two simulation results with $M_{\mathrm{A}}=3$ are shown: (i) the CME propagates perpendicular to the local magnetic field; and (ii) the CME propagates obliquely to the local magnetic field where only the case of the propagation angle of 80 degrees is shown. Also, from the first case it is shown that near the shock front the electron Bernstein waves are generated and can be converted to the electromagnetic waves (type II radio bursts) through direct linear mode conversion. It is found from the second case that near the shock front the electrostatic Langmuir waves (Z-mode) are generated and can be also converted to the electromagnetic waves through direct linear mode conversion. The generation of Z-modes was anticipated by Krauss-Varban (1989), and Bale et al. (1998) observed the generation of the transverse Z-mode waves, and not purely longitudinal Langmuir waves in the terrestrial electron foreshock.

Next we show the simulation results of the case of $M_{\mathrm{A}}=$ 1.5 and the propagation angle at 40 degrees. From other simulation results in which we changed the shock propagation angle from 90 to 40 degrees, we conclude that the Z-mode can be excited almost independent of the propagation angle and therefore the extra-ordinary electromagnetic waves are also emitted from the shock front through direct linear mode conversion.

This paper is organized as follows. In Sect. 2 the simulation model and the parameters used in the simulations are presented. In Sect. 3 we show the simulation results, and in Sect. 4 we summarize our results.

\section{Simulation model}

In Fig. 1 we present a schematic picture of the coronal mass ejections (CME) associated with a shock wave. We consider two models: (1) the CME propagates perpendicular to a magnetic field; and (2) the CME propagates obliquely with an 80 degree angle to a magnetic field. The lower figures in Fig. 1 show the system size and coordinate system used in the simulation.

We used 2D3V, the fully relativistic electromagnetic PIC code, modified from the 3D3V TRISTAN code (Buneman 1993). The system size is $L_{x}=800 \Delta$ and $L_{y}=10 \Delta$, where $\Delta(=1.0)$ is the grid size. The free boundary condition in the $x$ direction and the periodic boundary condition in the $y$ direction are imposed on particles and fields. We divided the simulation domain into two areas: left region $(x \leq 200 \Delta)$ and right region $(x>200 \Delta)$. In the left region, the average number of electron-ion pairs is 400 per cell, and the initial flow velocity $v=0.24 c$ is applied to the $x$-direction by the electric field $E_{z}$ through $E_{z}=v_{x} B_{y}$. This flow velocity is three times faster than Alfvén velocity $v_{\mathrm{A}}=0.08 c$. In the region on the right side, there are 100 electron-ion pairs per cell. The external magnetic field $B_{y}=B_{0}$ is applied to $y$-direction, that is, perpendicular to the flow direction.

The parameters used in this simulation are as follows. The ratio of ion mass to the electron mass is $m_{\mathrm{i}} / m_{\mathrm{e}}=64$, the
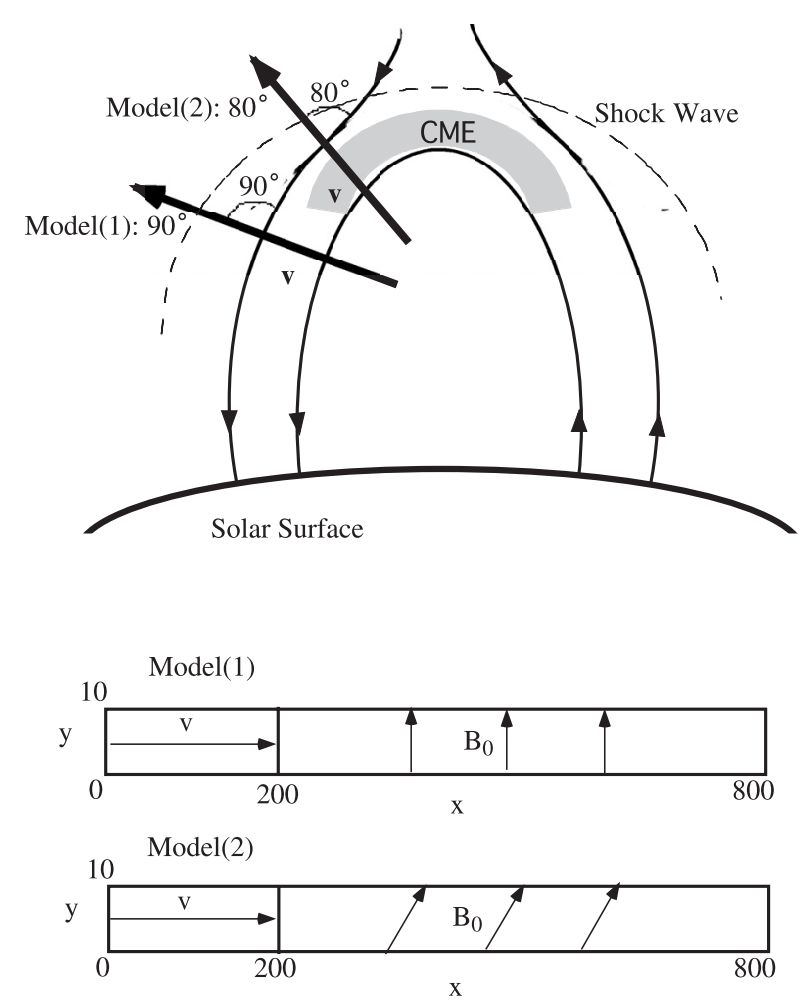

Fig. 1. Upper: schematic picture of coronal mass ejections (CME) associated with a shock wave and two models: Model (1) the CME propagates perpendicular to a magnetic field, and Model (2) the CME propagates obliquely with angle of 80 degree to a magnetic field. The lower figures show the system size and coordinate system used in the simulation.

ratio of electron cyclotron frequency to the plasma frequency is about $\omega_{\mathrm{ce}} / \omega_{\mathrm{pe}}=0.63$, plasma beta is $\beta=0.05$, skin depth is $c / \omega_{\text {pe }}=10.0 \Delta$, electron thermal velocity is $v_{\text {th,e }}=0.1 c$, electron Larmor radius is $\rho_{\mathrm{e}}=1.58 \Delta$, ion Larmor radius is $\rho_{\mathrm{i}}=12.7 \Delta$, and the simulation time step is $\omega_{\mathrm{pe}} \Delta t=0.05$, respectively. The parameters $\omega_{\text {ce }} / \omega_{\mathrm{pe}}, \beta, c / \omega_{\mathrm{pe}}, v_{\mathrm{th}, \mathrm{e}}, \rho_{\mathrm{e}}$, and $\rho_{\mathrm{i}}$ are calculated in the right region.

\section{Simulation results}

In Figs. 2 c-h we present summary of shock formation driven by the CME and the acceleration of both electrons and ions at $\omega_{\mathrm{pe}} t=90$. Figure $2 \mathrm{a}$ shows the electric field $E_{x}$ that is initially absent in the system. Figure $2 b$ show the initial magnetic field $B_{y}$ perpendicular to the flow direction of the CME. As the time goes on, the fast magnetosonic shock wave with Alfvén Mach $M_{\mathrm{A}}=3$ is formed as seen in Fig. $2 \mathrm{~d}$. The fast magnetosonic shock is characterized by a strong electrostatic field $E_{x}$ in the shock front as seen in Fig. 2c, which plays an important role in the particle acceleration through the surfatron mechanism (Sagdeev 1966; Sagdeev \& Shapiro 1973; Ohsawa 1985; Sakai \& Ohsawa 1987; Ohsawa \& Sakai 1987). Behind the shock front, there also appears an electrostatic field as seen in Fig. 2c, which is generated by the counter-streaming instability caused by the plasma particles reflected from the shock front. Figures $2 \mathrm{e}$ and $2 \mathrm{~g}$ show the phase-space plots $\left(V_{x}-x\right)$ for the ions and the electrons, respectively. As seen in Fig. 2e, 

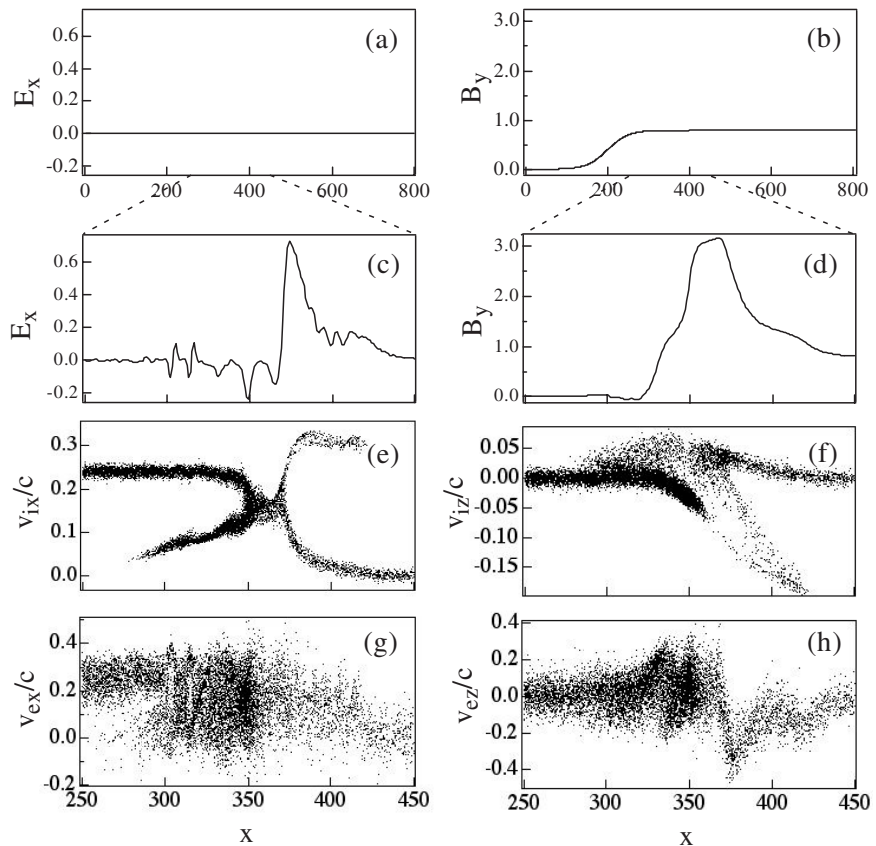

Fig. 2. Simulation results for Model (2). a) The electrostatic field $E_{x}$ and b) magnetic field $B_{y}$ at $\omega_{\mathrm{pe}} t=0$. c) The electrostatic field $E_{x}$ and d) magnetic field $B_{y}$ at $\omega_{\mathrm{pe}} t=90$. e) The ion phase space plot $\left(v_{\mathrm{i} x}-x\right)$ and f) ion phase space plot $\left(v_{\mathrm{i} z}-x\right)$ at $\omega_{\mathrm{pe}} t=90$. g) The electron phase space plot $\left(v_{\mathrm{e} x}-x\right)$ and $\left.\mathbf{h}\right)$ electron phase space plot $\left(v_{\mathrm{e} z}-x\right)$ at $\omega_{\mathrm{pe}} t=90$.

some ions are reflected to both sides of the shock front. While the reflected electrons behind the shock front mix together with the incoming electrons as seen in the electron phase-space plot of Fig. $2 \mathrm{~g}$, because the counter-streaming instability occurs in the shock region. This is why the electrostatic waves appear behind the shock front, as seen in Fig. 2c. We will investigate the nature of the electrostatic fields excited behind the shock front in detail later.

We note here that both electrons and ions can be strongly accelerated in the $z$-direction near the shock front through the surfatron mechanism. Figures $2 \mathrm{f}$ and $2 \mathrm{~h}$ show the phase-space plots $\left(v_{z}-x\right)$ of the ions and the electrons, respectively. The details of the acceleration process and the comparison with recent observations is discussed by Simnett et al. (2004), who show that the observed proton acceleration agrees well with the theory, as well as with the simulation results of the surfatron mechanism. The important simulation result for proton acceleration is that the surfatron acceleration mechanism can operate only in the propagation angles between 90 and 70 degrees.

Next we investigate the nature of the electrostatic waves excited behind the shock. Figure 3 shows dispersion relation and spatial distribution of both $E_{x}$ and $E_{z}$ for Model (1). Figure 3b shows the spatial distribution of $E_{x}$ at $\omega_{\mathrm{pe}} t=18$. We can see small oscillations behind the shock. To understand the characteristics of the electrostatic field behind the shock, we perform two-dimensional Fourier transformation: one-space and one-time for the first 512 steps $\left(\omega_{\mathrm{pe}} t=0\right.$ to $\left.\omega_{\mathrm{pe}} t=25.6\right)$ and $x=70$ to $x=326$, the region shown by the arrow in Fig. $3 \mathrm{~b}$. Then we obtained the dispersion relation of the electrostatic waves, as shown in Fig. 3a. The excited waves are shown by
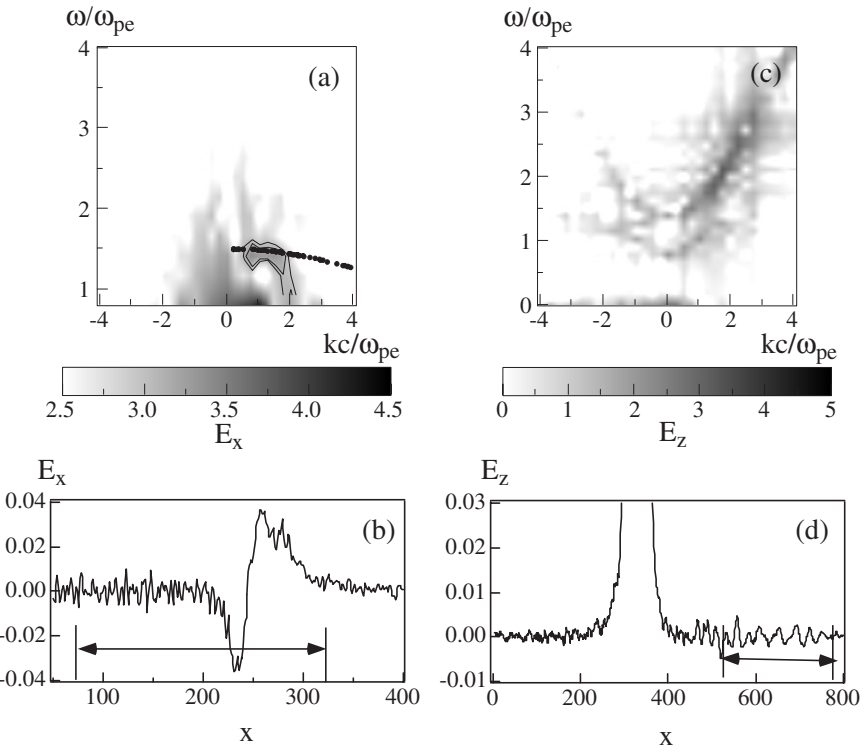

Fig. 3. Simulation results for Model (1): a) the dispersion relation for $E_{x}$ obtained by space-time Fourier transformation $(70 \leq x \leq 326$, and $\left.0 \leq \omega_{\text {pe }} t \leq 25\right)$. The dotted line shows the theoretical dispersion relation of the electron Bernstein wave. b) The snapshot of the $E_{x}$ at $\omega_{\mathrm{pe}} t=18$, where the spacial region to obtain the above dispersion relation is marked by the arrow. c) The dispersion relation for $E_{z}$ obtained by space-time Fourier transformation $(540 \leq x \leq 796$, and $\left.20 \leq \omega_{\mathrm{pe}} t \leq 71\right)$. d) The snapshot of the $E_{z}$ at $\omega_{\mathrm{pe}} t=66$, where the spacial region to obtain the above dispersion relation is marked by the arrow.

solid contour lines. The black dotted line shows the theoretical curve of the electron Bernstein mode, the theoretical curve obtained from the following dispersion equation (Stix 1992),

$$
1-\frac{2 \omega_{\mathrm{pe}}^{2}}{\lambda \omega_{\mathrm{ce}}^{2}} \mathrm{e}^{-\lambda} I_{1}(\lambda) \frac{\omega_{\mathrm{ce}}^{2}}{\omega^{2}-\omega_{\mathrm{ce}}^{2}}=0
$$

where $I_{1}$ is the modified Bessel function of the first order, and $\lambda=k^{2} v_{\mathrm{th}, \mathrm{e}}^{2} / \omega_{\mathrm{ce}}^{2}$. The electron Bernstein mode is defined by the parameters with the high density plasma where the Bernstein mode is excited: $\omega_{\text {ce }} / \omega_{\text {pe }}^{\prime}=0.63$ and $v_{\text {th,e }} / c=0.1$. Here $\omega_{\text {pe }}$ and $\omega_{\mathrm{pe}}^{\prime}$ are the plasma frequency in the low density and high density plasma, respectively. Their frequency ratio is 2.0 . The frequency and wave number in the dispersion relation are normalized by $\omega_{\text {pe }}$ and $\omega_{\text {pe }} / c$, respectively, where $\omega_{\text {pe }}$ is defined by using the electron density in the low density region. As seen in Fig. 3a, we conclude that the excited electrostatic waves are the electron Bernstein mode that may be generated by counterstreaming electrons behind the shock.

Figure $3 \mathrm{~d}$ shows a snapshot of the electric field $E_{z}$ at $\omega_{\text {pe }} t=66$. As seen in Fig. 3d (shown by an arrow), we find that the extra-ordinary electromagnetic waves (X-mode) are emitted from the shock front. To obtain the dispersion relation of the emitted X-mode, we perform a two-dimensional Fourier transformation: one-space and one-time for $\omega_{\mathrm{pe}} t=20$ to $\omega_{\mathrm{pe}} t=71$ and $x=540$ to $x=796$ (shown by the arrow in Fig. 3d). Then we obtained the dispersion relation of the Xmode, as seen in Fig. 3c. The frequency of the emitted X-mode is in the range $1.4 \omega_{\mathrm{pe}}$ and about $3.5 \omega_{\mathrm{pe}}$. The emission mechanism of the $\mathrm{X}$-mode is due to the direct linear mode conversion 

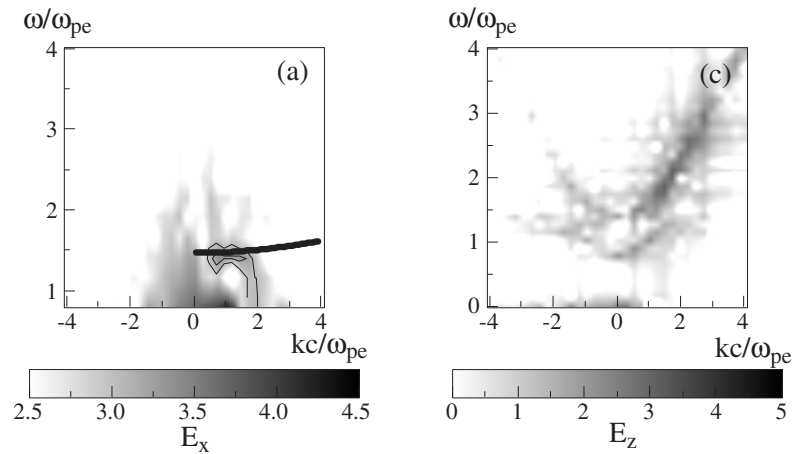

$\mathrm{E}_{\mathrm{X}}$

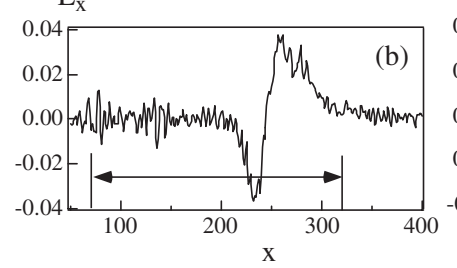

$\mathrm{E}_{\mathrm{z}}$

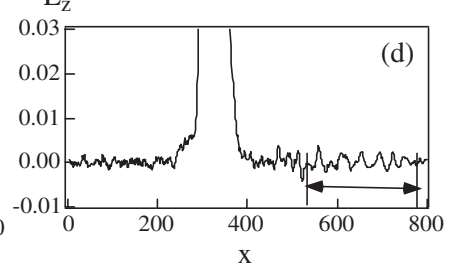

Fig. 4. Simulation results for Model (2): a) the dispersion relation for $E_{x}$ obtained by space-time Fourier transformation (70 $\leq x \leq 326$, and $\left.0 \leq \omega_{\text {pe }} t \leq 25\right)$. The dotted line shows the theoretical dispersion relation of the Langmuir wave (z-mode). b) The snapshot of the $E_{x}$ at $\omega_{\mathrm{pe}} t=18$, where the spacial region to obtain the above dispersion relation is marked by the arrow. c) The dispersion relation for $E_{z}$ obtained by space-time Fourier transformation $(540 \leq x \leq 796$, and $\left.20 \leq \omega_{\mathrm{pe}} t \leq 71\right)$. d) The snapshot of the $E_{z}$ at $\omega_{\mathrm{pe}} t=66$, where the spacial region to obtain the above dispersion relation is marked by the arrow.

(Budden 1961) from the electron Bernstein mode due to the density gradient near the shock front.

Next we added external magnetic field $B_{x}$ and we investigated the effect of the oblique propagation of shocks on the wave emission from the shock front. We changed the propagation angle from 90 to 40 degrees. The electromagnetic wave emission was observed in the above angles. Here we present the simulation result for the propagation angle of 80 degrees (Model (2)). Figure 4 shows dispersion relation and spatial distribution of both $E_{x}$ and $E_{z}$ for Model (2). Figure $4 \mathrm{~b}$ shows the spatial distribution of $E_{x}$ at $\omega_{\text {pe }} t=18$. We can see small oscillations behind the shock. To understand the characteristics of the electrostatic field behind the shock, we performed twodimensional Fourier transformation: one-space and one-time for the first 512 steps $\left(\omega_{\mathrm{pe}} t=0\right.$ to $\left.\omega_{\mathrm{pe}} t=25\right)$ and $x=70$ to $x=326$ (shown by the arrow in Fig. 4b). Then we obtained the dispersion relation of the electrostatic waves, as shown in Fig. 4a. The excited waves are shown by solid contour lines. The black dot line shows the theoretical curve of the obliquely propagating Langmuir wave (Z-mode), theoretical curve obtained from the following dispersion equation

$\omega^{2}=\omega_{\mathrm{pe}}^{2}+3 k^{2} v_{\text {th }, \mathrm{e}}^{2}+\omega_{\mathrm{ce}}^{2} \sin ^{2} \theta$,

where $v_{\text {th,e }}$ is the electron thermal velocity and $\theta$ the angle between the wave-vector and the magnetic field. We used $v_{\mathrm{th}, \mathrm{e}}=$ $0.1 c$ and $\theta=4 \pi / 9$. As seen in Fig. $4 a$, we conclude that the excited electrostatic waves are the Z-mode that may be generated by counter-streaming electrons behind the shock.
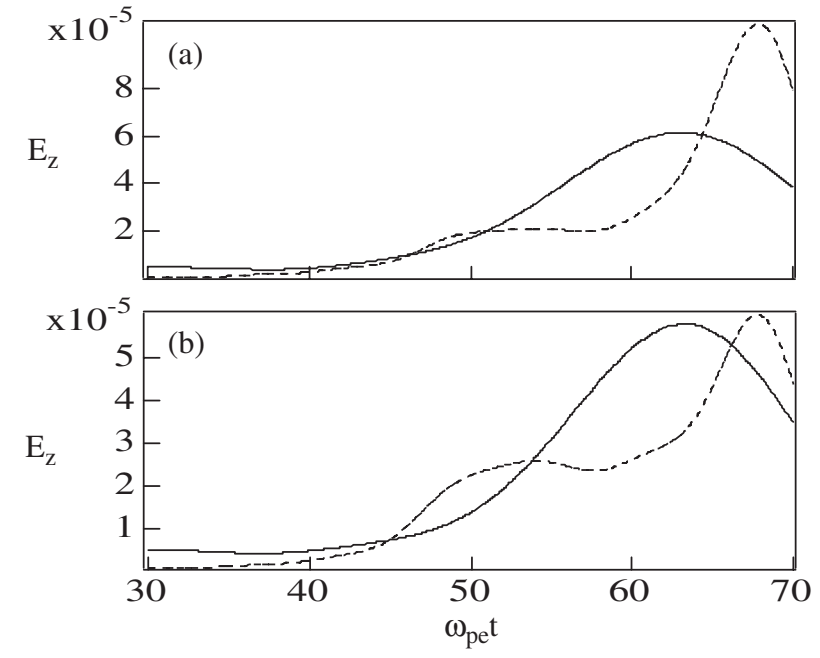

Fig. 5. Time history of $E_{z}$ for Model (1)-a), and Model (2)-b), obtained from the Inverse Fourier transformation using the data of Figs. 3c and $4 \mathrm{c}$. The solid line is obtained by using data of $1.5 \leq \omega / \omega_{\mathrm{pe}} \leq 2.0$ and $0 \leq k c / \omega_{\mathrm{pe}} \leq 3.0$, and the dashed line is obtained by using data of $2.5 \leq \omega / \omega_{\mathrm{pe}} \leq 3.5$ and $0 \leq k c / \omega_{\mathrm{pe}} \leq 4.0$.

Figure $4 \mathrm{~d}$ shows a snapshot of the electric field $E_{z}$ at $\omega_{\text {pe }} t=66$. As seen in Fig. 4d (see arrow), we find that the extraordinary electromagnetic waves (X-mode) are emitted from the shock front. To obtain the dispersion relation of the emitted $\mathrm{X}$-mode, we performed two-dimensional Fourier transformation: one-space and one-time for $\omega_{\mathrm{pe}} t=20$ to $\omega_{\mathrm{pe}} t=71$ and $x=540$ to $x=796$ (arrow in Fig. 4d). Then we obtained the dispersion relation of the X-mode, as seen in Fig. 4c. The frequency of the emitted X-mode ranges between $1.4 \omega_{\mathrm{pe}}$ and about $3.2 \omega_{\text {pe }}$. The emission mechanism of the X-mode is due to the direct linear mode conversion from the $\mathrm{Z}$-mode due to the density gradient near the shock front.

To see the timing of emission of the fundamental and second harmonic, we present the time history of $E_{z}$ for Model (1) (in Fig. 5a), and Model (2) (in Fig. 5b), which are obtained from the Inverse Fourier transformation using the data in Figs. $3 \mathrm{c}$ and $4 \mathrm{c}$, respectively. The solid line showing the fundamental emission is obtained by using data for $1.5 \leq \omega / \omega_{\text {pe }} \leq$ 2.0 and $0 \leq k c / \omega_{\text {pe }} \leq 3.0$, and the dashed line showing the second harmonic is obtained by using data for $2.5 \leq \omega / \omega_{\mathrm{pe}} \leq 3.5$ and $0 \leq k c / \omega_{\text {pe }} \leq 4.0$. As seen in Fig. 5, the second harmonic emission is slightly delayed from the fundamental emission for both cases.

Here we note the shock speed for the propagation angle with 70 degrees that can be estimated from the moving positions of the maximum value of the magnetic field $B_{y}$. Figures $6 \mathrm{a}$ and $6 \mathrm{~b}$ show the time evolution of shock speeds, $M_{\mathrm{A}}=3.0$ and $M_{\mathrm{A}}=1.5$, respectively.

From other simulation results in which we changed the shock propagation angle from 90 to 40 degrees, we conclude that the Z-mode can be excited with the amplitude the same as the case presented here and therefore the extra-ordinary electromagnetic waves are also emitted from the shock front the same way as presented here. In Fig. 7 we show the simulation results of the case of $M_{\mathrm{A}}=1.5$ and the propagation angle with 

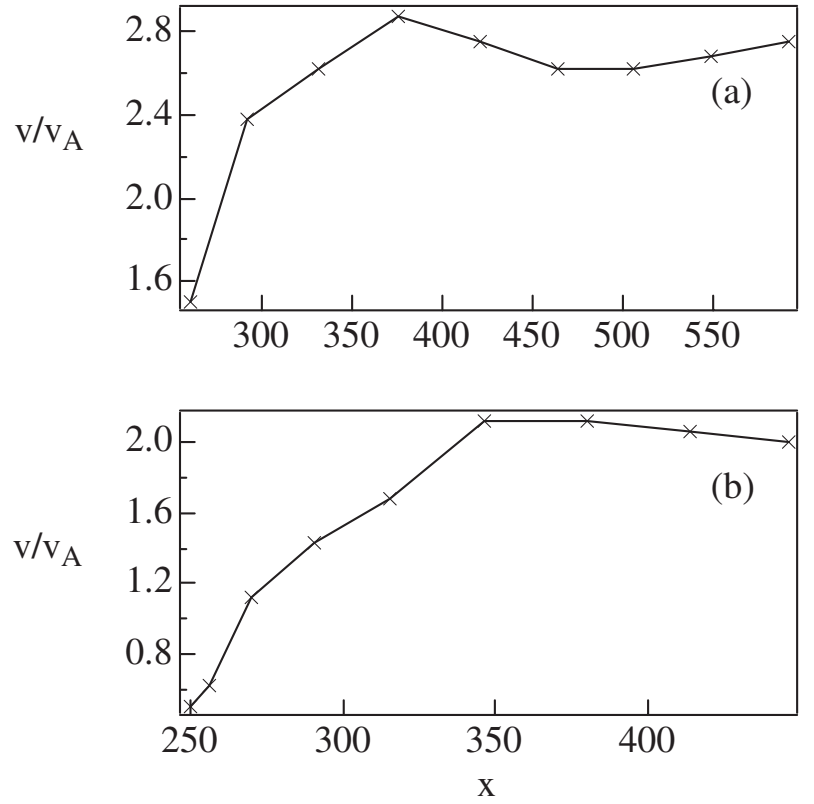

Fig. 6. The time evolution of shock speeds for the case of 70 degrees estimated from the moving positions of the maximum value of $B_{y}: \mathbf{a}$ ) $\left.M_{\mathrm{A}}=3.0, \mathbf{b}\right) M_{\mathrm{A}}=1.5$.
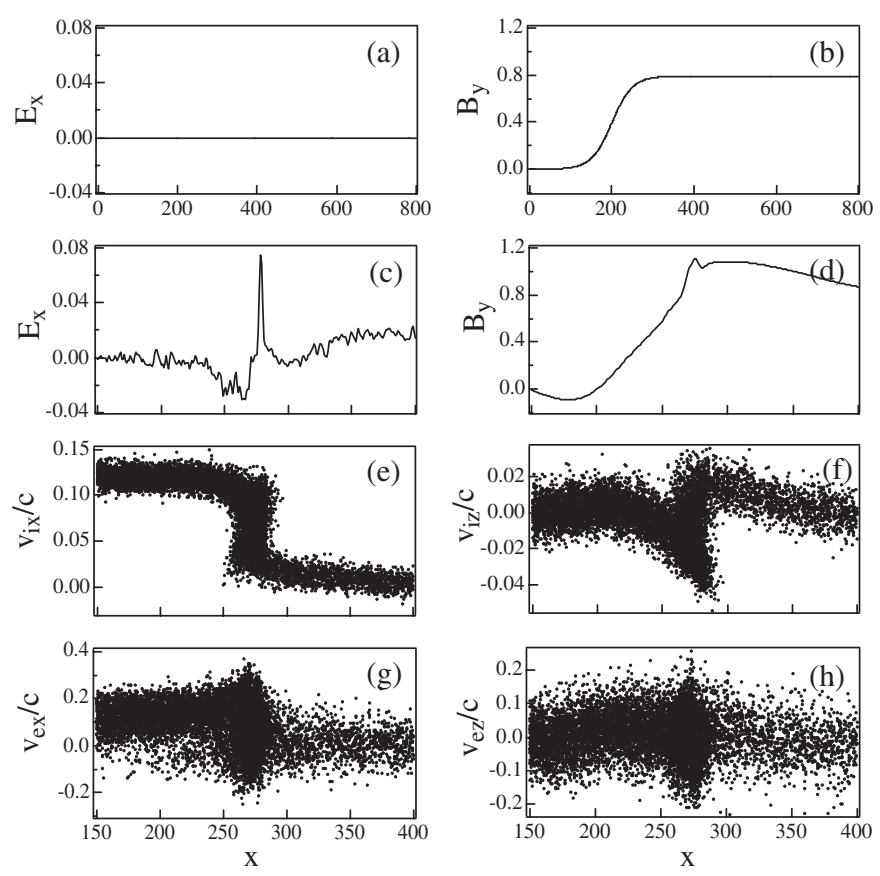

Fig. 7. Simulation results for the case of $M_{\mathrm{A}}=1.5$ and the propagation angle with 40 degrees. a) The electrostatic field $E_{x}$ and b) magnetic field $B_{y}$ at $\omega_{\text {pe }} t=0$. c) The electrostatic field $E_{x}$ and d) magnetic field $B_{y}$ at $\omega_{\mathrm{pe}} t=90$. e) The ion phase space plot $\left(v_{\mathrm{i} x}-x\right)$ and $\left.\mathbf{f}\right)$ ion phase space plot $\left(v_{\mathrm{i} z}-x\right)$ at $\omega_{\mathrm{pe}} t=90$. g) The electron phase space plot $\left(v_{\mathrm{e} x}-x\right)$ and $\left.\mathbf{h}\right)$ electron phase space plot $\left(v_{\mathrm{e} z}-x\right)$ at $\omega_{\mathrm{pe}} t=90$.

40 degrees. Figure $7 \mathrm{a}$ shows the electric field $E_{x}$ that is initially absent in the system, and Fig. 7b shows the initial magnetic field $B_{y}$ perpendicular to the flow direction of the CME. As time goes on, the weak fast magnetosonic shock wave with Alfvén Mach $M_{\mathrm{A}}=1.5$ is formed as seen in Fig. $7 \mathrm{~d}$. The weak fast magnetosonic shock is characterized by a weak
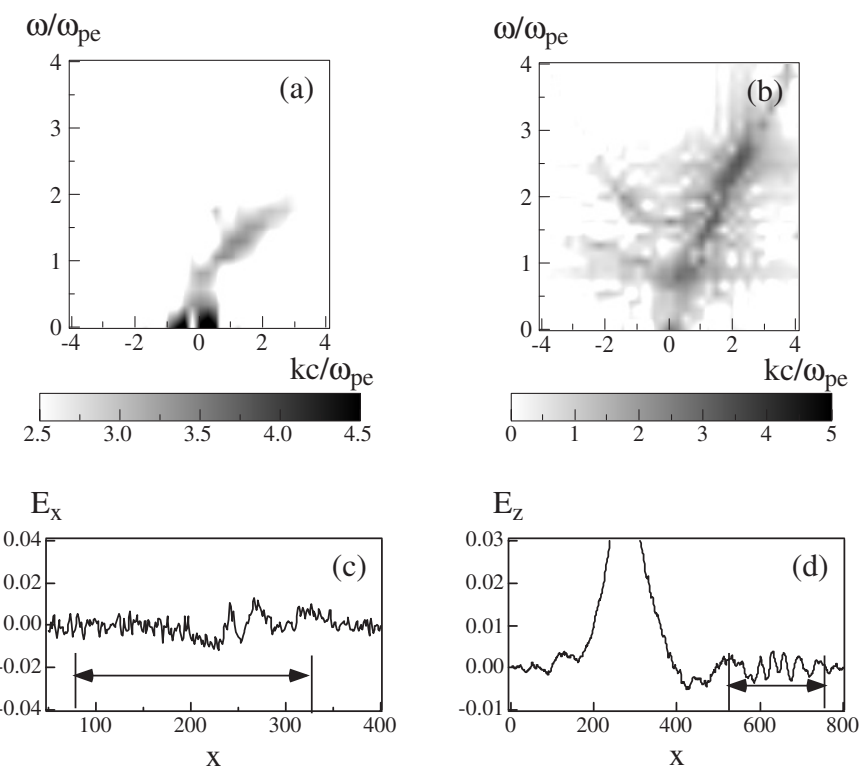

Fig. 8. Simulation results for the case of $M_{\mathrm{A}}=1.5$ and the propagation angle with 40 degrees: a) The dispersion relation for $E_{x}$ obtained by space-time Fourier transformation $\left(70 \leq x \leq 326\right.$, and $\left.0 \leq \omega_{\mathrm{pe}} t \leq 25\right)$. b) The snapshot of the $E_{x}$ at $\omega_{\mathrm{pe}} t=18$, where the spacial region to obtain the above dispersion relation is marked by the arrow. c) The dispersion relation for $E_{z}$ obtained by space-time Fourier transformation $\left(540 \leq x \leq 796\right.$, and $\left.20 \leq \omega_{\mathrm{pe}} t \leq 71\right)$. d) The snapshot of the $E_{z}$ at $\omega_{\mathrm{pe}} t=66$, where the spacial region to obtain the above dispersion relation is marked by the arrow.

electrostatic field $E_{x}$ in the shock front as seen in Fig. 7c, which contrasts with the strong shock case seen in Fig. 2c. Behind the shock front, an electrostatic field also appears as seen in Fig. 7c, which is generated by the counter-streaming instability caused by the plasma particles reflected from the shock front. Figures $7 \mathrm{e}$ and $7 \mathrm{~g}$ show the phase-space plots $\left(V_{x}-x\right)$ for the ions and the electrons, respectively. As seen in Fig. 7e, ions are not reflected to both sides of the shock front as in the strong shock (see Fig. 2e). While, as seen in the electron phasespace plot of Fig. $7 \mathrm{~g}$, the reflected electrons behind the shock front mix with the incoming electrons, because the counterstreaming instability occurs in the shock region. This is why the electrostatic waves appear behind the shock front (Fig. 7c).

Here we note that both electrons and ions cannot be accelerated in the $z$-direction near the shock front through the surfatron mechanism. Figures $7 \mathrm{f}$ and $7 \mathrm{~h}$ show the phase-space plots $\left(v_{z}-x\right)$ of the ions and the electrons, respectively. The important simulation result for proton acceleration is that the surfatron acceleration mechanism can operate only in the propagation angles between 90 and 70 degrees.

Figure 8 shows dispersion relation and spatial distribution of both $E_{x}$ and $E_{z}$ for the case of $M_{\mathrm{A}}=1.5$ and the propagation angle with 40 degrees. Figure $8 \mathrm{c}$ shows the spatial distribution of $E_{x}$ at $\omega_{\mathrm{pe}} t=18$. We can see small oscillations behind the shock. To understand the characteristics of the electrostatic field behind the shock, we performed two-dimensional Fourier transformation: one-space and one-time for the first 512 steps $\left(\omega_{\mathrm{pe}} t=0\right.$ to $\left.\omega_{\mathrm{pe}} t=25\right)$ and $x=70$ to $x=326$ (region shown by the arrow in Fig. 8c). Then we obtained the dispersion relation 
of the electrostatic waves, as shown in Fig. 8a. We conclude that the excited electrostatic waves are the Z-mode that may be generated by counter-streaming electrons behind the shock.

Figure 8d shows a snapshot of the electric field $E_{z}$ at $\omega_{\text {pe }} t=66$. As seen in Fig. 8d (see arrow), we find that the extraordinary electromagnetic waves (X-mode) are emitted from the shock front. To obtain the dispersion relation of the emitted $\mathrm{X}$-mode, we performed two-dimensional Fourier transformation: one-space and one-time for $\omega_{\text {pe }} t=20$ to $\omega_{\text {pe }} t=71$ and $x=540$ to $x=796$ (arrow in Fig. 8d). Then we obtained the dispersion relation of the X-mode, as seen in Fig. 8b. The frequency of the emitted X-mode ranges between $1.4 \omega_{\text {pe }}$ and about $3.2 \omega_{\mathrm{pe}}$. The emission mechanism of the $\mathrm{X}$-mode is the direct linear mode conversion from the Z-mode due to the density gradient near the shock front.

\section{Summary}

We investigated the wave emission process of solar type II radio bursts associated with Coronal Mass Ejections (CME) by using two dimensional, electromagnetic, relativistic ParticleIn-Cell (PIC) code. We did many simulations by changing the shock Alfvén Mach number from $M_{\mathrm{A}}=1.5$ to $M_{\mathrm{A}}=3.0$ and also by changing the propagation angle of the shock from 90 to 40 degrees. It was shown that for fast magnetosonic shock waves with $M_{\mathrm{A}} \geq 2.0$ and the propagation angle between 90 and 70 degrees protons near the shock front can be accelerated by the surfatron acceleration mechanism. The emission of the electromagnetic waves from shock front was observed almost independently of the Alfvén Mach and the propagation angles. We only show two simulations here: (i) the CME propagates perpendicular to the local magnetic field; and (ii) the CME propagates obliquely to the local magnetic field where only the case of the propagation angle of 80 degrees is shown. It was shown that fast magnetosonic shock wave is formed with both protons and electrons accelerated by the surfatron acceleration mechanism. It was also shown from the first case that near the shock front the electron Bernstein waves are generated and can be converted into the extra-ordinary electromagnetic waves (type II radio bursts) through direct linear mode conversion. It was found from the second case that near the shock front the electrostatic Langmuir waves (Z-mode) are generated and can be also converted to the extra-ordinary electromagnetic waves through direct linear mode conversion.
Acknowledgements. The authors thank the anonymous referee for very constructive comments that improved our original paper. The authors thank Dr. H. Aurass for discussion of observational results of solar type II radio bursts. S. Saito is supported by a research fellowship of the Japan Society for the Promotion of Science (JSPS) for young scientists, as JSPS Research Fellow.

\section{References}

Aschwanden, M. J. 2004, in Physics of The Solar Corona (SpringerVerlag), Chap. 15, 657

Bale, S. D., Kellogg, P. J., Goetz, K., \& Monson, S. J. 1998, Geophys. Res. Lett., 25(1), 9

Bale, S. D., Larson, D. E., Lin, R. P., et al. 2000, J. Geophys. Res., 105(A12), 27353

Bell, A. R. 1978, MNRAS, 182, 147

Blandford, R. D., \& Ostriker, J. P. 1978, ApJ, 221, L29

Budden, K. G. 1961, Radio Waves in the Ionosphere (New York: Cambridge Univ. Press)

Bulanov, S. V., \& Sokolov, I. V. 1984, Soviet Astro. Lett., 10, 247

Buneman, O. 1993, in Computer Space Plasma Physics, Simulation Techniques and Software, ed. H. Matsumoto, \& Y. Omura (Tokyo: Terra Scientific), 67

Cairns, I. H., Knock, S. A., Robinson P. A., \& Kuncic, Z. 2003, Space Sci. Rev., 107, 27

Fermi, E. 1949, Phys. Rev., 75, 1169

Goldman, M. V., \& Smith, D. F. 1986, in Physics of the SUN, ed. P. A. Sturrock, T. E. Holzer, D. M. Mihalas, \& R. K. Ulrich (D. Reidel Publ. Co.), Vol. 2, Chap. 15, 325

Krauss-Varban, D. 1989, J. Geophys. Res., 94, 3527

Lee, M. A. 1983, J. Geophys. Res., 88, 6109

Melrose, D. B. 1980, Aust. J. Phys., 33, 121

Ohsawa, Y. 1985, Phys. Fluids, 28, 2130

Ohsawa, Y., \& Sakai, J. I. 1987, ApJ, 313, 440

Reames, D. Y. 1999, Space Sci. Rev., 90, 413

Reames, D. Y. 2002, ApJ, 571, L63

Sagdeev, R. Z. 1966, Reviews of Plasma Physics, ed. M. A. Leontovich (New York: Consultants Bureau), 4, 23

Sagdeev, R. Z., \& Shapiro, V. D. 1973, JETP Lett., 17, 279

Sakai, J. I., \& Ohsawa, Y. 1987, Space Sci. Rev., 46, 113

Simnett, G. M. 2003, Sol. Phys., 213, 387

Simnett, G. M. 1, Sakai, J. I., \& Forsyth, R. J. 2003, A\&A, submitted

Smith, D. F. 1970, Adv. Astro. Astrophys., 7, 147

Sokolov, I. V., Roussev, I. I., Gombosi, T. I., et al. 2004, ApJ, 616, L171

Stix, T. H. 1992, Waves in Plasmas (New York: AIP) 